\title{
The MADS transcription factor CmANR1 positively modulates root system development by directly regulating CmPIN2 in chrysanthemum
}

\author{
Cui-Hui Sun ${ }^{1}$, Jian-Qiang Yu', Xi Duan², Jia-Hui Wang ${ }^{1}$, Quan-Yan Zhang', \\ Kai-Di Gu', Da-Gang Hu and Cheng-Shu Zheng ${ }^{1}$
}

\begin{abstract}
Plant root systems are essential for many physiological processes, including water and nutrient absorption. MADS-box transcription factor (TF) genes have been characterized as the important regulators of root development in plants; however, the underlying mechanism is largely unknown, including chrysanthemum. Here, it was found that the overexpression of CMANR1, a chrysanthemum MADS-box TF gene, promoted both adventitious root (AR) and lateral root (LR) development in chrysanthemum. Whole transcriptome sequencing analysis revealed a series of differentially expressed unigenes (DEGs) in the roots of CMANR1-transgenic chrysanthemum plants compared to wild-type plants. Functional annotation of these DEGs by alignment with Gene Ontology (GO) terms and biochemical pathway Kyoto Encyclopedia of Genes and Genomes (KEGG) enrichment analysis indicated that CmANR1 TF exhibited "DNA binding" and "catalytic" activity, as well as participated in "phytohormone signal transduction". Both chromatin immunoprecipitation-polymerase chain reaction (ChIP-PCR) and gel electrophoresis mobility shift assays (EMSA) indicated the direct binding of CMPIN2 to the recognition site CArG-box motif by CmANR1. Finally, a firefly luciferase imaging assay demonstrated the transcriptional activation of CMPIN2 by CmANR1 in vivo. Overall, our results provide novel insights into the mechanisms of MADS-box TF CmANR1 modulation of both AR and LR development, which occurs by directly regulating auxin transport gene CMPIN2 in chrysanthemum.
\end{abstract}

\section{Highlight}

MADS-box TF CmANR1 modulates both AR and LR development by directly regulating auxin transport gene CmPIN2 in chrysanthemum.

Correspondence: Da-Gang Hu (fap_296566@163.com) or Cheng-Shu Zheng (zcs@sdau.edu.cn)

${ }^{1}$ National Key Laboratory of Crop Biology, College of Horticulture Science and Engineering, Shandong Agricultural University, Tai-An, Shandong 271018

China

${ }^{2}$ Shandong Agricultural and Engineering University, Ji-Nan, Shandong, China These authors contributed equally: Da-Gang Hu, Cheng-Shu Zheng

\section{Introduction}

Plant roots are crucial to their anchorage, absorption of nutrients and water, as well as to establishment of beneficial symbioses with the surrounding soil microorganism communities $^{1,2}$. In some cases, such as in the dicot model plant Arabidopsis, the radicle is generated during embryogenesis within a seed. Following germination, the radicle elongates as the primary root (PR), and typically

\section{(c) The Author(s) 2018}

(c) (i) Open Access This article is licensed under a Creative Commons Attribution 4.0 International License, which permits use, sharing, adaptation, distribution and reproduction cc) in any medium or format, as long as you give appropriate credit to the original author(s) and the source, provide a link to the Creative Commons license, and indicate if changes were made. The images or other third party material in this article are included in the article's Creative Commons license, unless indicated otherwise in a credit line to the material. If material is not included in the article's Creative Commons license and your intended use is not permitted by statutory regulation or exceeds the permitted use, you will need to obtain permission directly from the copyright holder. To view a copy of this license, visit http://creativecommons.org/licenses/by/4.0/. 
grows into a central taproot together with the sequential development of the associated lateral roots $(\mathrm{LRs})^{3}$. However, monocot plants such as rice (Oryza sativa) and maize (Zea mays) develop a more complicated root system ${ }^{4}$. Apart from a specific embryonic PR and several seminal roots (SRs) in maize, most cereals possess an expanding shoot-borne root system ${ }^{5}$. The postembryonic shoot-born roots, called brace roots (BRs) and crown roots (CRs), are also able to branch developing lateral roots $^{6}$. Moreover, some plants species such as chrysanthemum (Chrysanthemum morifolium), African violet (Saintpaulia spp.), strawberries (Fragaria spp.), and garlic (Allium sativum), which propagate vegetatively, firstly develop numerous adventitious roots (ARs) from the basal cuttings or stolons in their typical ecological environments $^{3}$. Successively, LRs or higher-order LRs originate from the existing ARs to expand the root system in order to obtain more water and nutrients.

Adventitious roots, which are similar to lateral roots, develop post-embryonically. ARs usually arise from vegetative organs, such as the stolons, rhizomes, leaves, and stems, while LRs often originate from existing roots, such as the PRs, previous LRs, or $\mathrm{ARs}^{3}$. Despite the differences in origin, the formation and development of ARs and LRs is controlled by a suite of similar endogenous and environmental factors ${ }^{3,7,8}$. Among these common regulatory factors, auxin is the most vital regulator of both AR and LR development ${ }^{9-13}$. Natural auxins (e.g., indole3-acetic acid (IAA)) and synthetic analogs (e.g., indole-3butyric acid (IBA)) have a powerful and stimulatory effect on rooting in many plant species ${ }^{12,14,15}$. A diverse range of studies have shown that auxin is central to AR and LR development in plants, where it cross-talks with other signals (e.g., calcium signal) ${ }^{16,17}$, regulatory genes (e.g., AtMYB93, SHR, and ERF3) ${ }^{18-20}$, or phytohormones (e.g., ethylene and gibberellins) $)^{21-23}$. Furthermore, auxinrelated biological processes, such as signal transduction, polar transport, and local biosynthesis, account for the primary underlying molecular mechanisms regulating AR or LR formation ${ }^{10,11,24-27}$.

Generally, auxin is produced in the aerial tissues (such as apical meristems) and is then distributed locally and systemically throughout whole plant via two distinct yet interconnected ways: a direct and fast flow from shoots to roots via the vascular central cylinder, and cell-to-cell active polar transport through the outer layers of the root cells $^{28,29}$. However, auxin is also synthesized in the root tips, where auxin transport is characterized by dual polarities. In the roots, auxin polar transport has been described as acropetally (towards root apex) and basipetally (from apex to base) ${ }^{30}$. The auxin efflux carriers PINFORMED (PIN) proteins as well as auxin influx carriers AUXIN RESISTANT1/LIKE AUX1 (AUX1/LAX) proteins have been identified as the main components responsible for auxin transport ${ }^{31,32}$. The asymmetric subcellular distribution and localization of carrier proteins contributes to their polarity ${ }^{33}$, among which PIN polarity has been shown to be the primary direction-determining factor in auxin polar transport ${ }^{34}$. So far, PIN members have mostly been well studied in Arabidopsis where they exhibit unique but somewhat overlapped localization in various cell types. PIN1 localizes in the basal end of the vascular cells, facilitating the root-ward movement of auxin $^{35}$. PIN2 predominantly resides basally in cortical cells, and apically in the epidermal and root cap cells ${ }^{34,36}$. PIN3 has been detected in the columella cells of the roots in an apolar manner. PIN7 localizes basally in the stele cells in the meristem and elongation zone ${ }^{37}$. Recent studies reported that PIN5, PIN6, and PIN8 located on both the endoplasmic reticulum and plasma membrane ${ }^{38-42}$. Additionally, PINs have been functionally identified as vital regulators of numerous auxin-related developmental processes. For instance, OsPIN1 takes part in auxindependent AR emergence and development in rice ${ }^{43}$. OsPIN2 regulates tiller angle, number, as well as plant height by enhancing basipetal auxin transport in rice ${ }^{44}$. In Arabidopsis, the triple mutant pin1 pin 3 pin4 is defective in PR development ${ }^{33}$; PIN6 is required for LR and AR organogenesis by controlling auxin homeostasis and distribution $^{42}$; and PIN8 exerts a crucial role on pollen development and functionality ${ }^{38,39}$.

Transcription factors (TFs) represent a large portion of the essential regulators of many developmental aspects in plants. With respect to root development regulation, a multitude of TFs have been reported to participate in, and influence, a diverse set of developmental stages of different root types in many plant species ${ }^{18-20,45,46}$. The MADS-box TFs became a point of interest into the genetic regulation of root development. In addition to developmental regulation of flower, fruit, seed, and leaf developmental regulation in plants ${ }^{47-50}$, an increasing number of MADS-box genes have been reported to be involved in root system development. For example, AtANR1 was the first MADS-box TF gene identified to regulate LR elongation under heterogeneous nitrate conditions $^{51}$. The MADS-box gene XAL1/AGL12 gene was determined to be necessary for normal root development and growth via a positive control on cell cycle components $^{52}$. XAL2/AGL14 plays an essential role in robust root patterning by modulating auxin polar transport $^{53}$. AGL21 was found to be a positive regulator of LR initiation and growth by increasing local auxin biosynthesis in Arabidopsis ${ }^{27}$. The ANR1-like gene OsMADS25 positively regulates both $\mathrm{PR}$ and LR development by promoting nitrate accumulation in rice ${ }^{54}$. GmNMHC5, a MADS-box TF gene in soybean, promotes LR development in a sucrose-dependent manner ${ }^{55}$. 
Recently, it was reported that the ectopic expression of CmANR1/CmAGL44 in Arabidopsis could promote LR development ${ }^{56}$. However, the underlying mechanisms of CmANR1 modulation of LR development are largely unknown. Here, it was found that CmANR1 positively modulates AR and LR development by directly regulating CmPIN2 in chrysanthemum. The potential application of the CmANR1 gene in controlling root system development and its theoretical research value in breeding programs in chrysanthemum are discussed in this paper.

\section{Materials and methods}

\section{Chrysanthemum and growth conditions}

The 35S::CmANR1-1258 (green fluorescent protein (GFP) tag) overexpressed vector was constructed as previously described ${ }^{56}$. It was then transformed into Agrobacterium strain GV3101. The wild-type (WT) tissuecultured chrysanthemum were kindly provided by Professor Gao (China Agricultural University). The CmANR1-transgenic chrysanthemum were obtained by Agrobacterium-mediated transformation of leaf discs ${ }^{57}$. In tissue-cultured condition, CmANR1-transgenic and WT plants were cultivated in vitro on Murashige and Skoog (MS) medium in the standardized culture room.

In hydroponic-cultured condition, the chrysanthemum were cultivated in improved Hogland nutrient solution $\left(\mathrm{CaCl}_{2} 555 \mathrm{mg} / \mathrm{L}, \quad \mathrm{MgSO}_{4} \cdot 7 \mathrm{H}_{2} \mathrm{O} \quad 493 \mathrm{mg} / \mathrm{L}, \quad \mathrm{KH}_{2} \mathrm{PO}_{4}\right.$ $136 \mathrm{mg} / \mathrm{L}, \quad \mathrm{FeSO} 4.7 \mathrm{H}_{2} \mathrm{O} \quad 27.6 \mathrm{mg} / \mathrm{L}, \quad$ EDTA-2Na $3.73 \mathrm{mg} / \mathrm{L}, \quad \mathrm{KNO}_{3} \quad 10 \mathrm{mM}, \quad \mathrm{H}_{3} \mathrm{BO}_{4} \quad 2.86 \mathrm{mg} / \mathrm{L}$, $\mathrm{MnCl}_{2} \cdot 4 \mathrm{H}_{2} \mathrm{O} \quad 1.82 \mathrm{mg} / \mathrm{L}, \quad \mathrm{ZnSO}_{4} \cdot 7 \mathrm{H}_{2} \mathrm{O} \quad 0.23 \mathrm{mg} / \mathrm{L}$, $\mathrm{H}_{2} \mathrm{MoO}_{4} \cdot \mathrm{H}_{2} \mathrm{O} 0.09 \mathrm{mg} / \mathrm{L}, \mathrm{CuSO}_{4} \cdot 5 \mathrm{H}_{2} \mathrm{O} 0.08 \mathrm{mg} / \mathrm{L}, \mathrm{pH}=$ 5.6). A simple aeration device was used to supply oxygen in hydroponic condition in case that roots would go rotted.

\section{Arabidopsis AR rooting assays}

The Arabidopsis lines used here were the CmANR1overexpressing (OE) lines and the "Columbia" ecotype. For the procedure of seed sterilization and the growth conditions of Arabidopsis, refer to our previous study ${ }^{56}$. After sterilization, the seeds were planted on MS medium with $1 \%(\mathrm{w} / \mathrm{v})$ sucrose and $0.7 \%(\mathrm{w} / \mathrm{v})$ agar. Then, the seedlings with only two cotyledons on were trimmed, leaving only the hypocotyls. The hypocotyls were transferred vertically on either MS or MS added with $0.1 \mu \mathrm{M}$ IBA for AR rooting analysis. The AR rooting assays were performed in a dark growth incubator $\left(23 \pm 1{ }^{\circ} \mathrm{C}, 40 \%\right.$ relative humidity) for a week.

\section{Morphological characterization of roots in chrysanthemum and Arabidopsis}

The 20-day-old in vitro chrysanthemum and 40-day-old hydroponic-cultured chrysanthemum were used for root morphological characterization. The relevant root data such as root total length, root volume, and root surface were analyzed by WinRHIZO software (Regent Instruments Inc., Canada). The root numbers of AR and LR were counted using Image J software (NIH, Bethesda, MD, USA) of digital images of roots. The root morphology of CmANR1-overexpressing and WT control Arabidopsis seedlings was on observed MS solid medium with $0.8 \%$ agar. Photos of the seedlings were taken after 1 week of darkness, about 10 days old. AR number and AR length was measured by hand using Image J software (NIH, Bethesda, MD, USA).

\section{RNA-Seq data processing, de novo assembly, and annotation}

The samples were the 40-day-old hydroponiccultured CmANR1-overexpressing plants (CmANR1OVX56, -OVX67, -OVX81) and WT plants. Three independent plants of each line consisted of the triplicate samples. Total RNAs of the samples were extracted using the RNeasy plant mini kit (New England Biolabs Inc., New England). The complementary DNA (cDNA) library was prepared as described by Grabherr et al. ${ }^{58}$ and the sequencing was performed on the Illumina HiSeq Platform (Ori-gene Inc., Beijing, China).

Raw RNA-Sequencing (RNA-Seq) reads were conducted with Cutadapt based on BMA algorithm to remove sequence artifacts such as adapter sequences on both ends, low-quality trailing $\left(\mathrm{Q}_{30}\right)$, 3'-end barcode sequences, and reads with lengths less than $60 \mathrm{bp}$. The remaining valid cleaned reads were processed into de novo transcript assembly according to a previous study ${ }^{58}$. Furthermore, the resulting reads were assembled using iAssembler with a threshold of $(-p)$ set to $99^{59,60}$.

The resulting unigenes were screened by BLAST (Basic Local Alignment Search Tool) against the GenBank nonredundant (NR), TrEMBL, Swiss-Prot, Pfam, and KOG. The unigenes with a cutoff of $E$-value of $\leq 1 \mathrm{e}-5$ and $\geq 30 \%$ identify were needed for further functional annotation. The chrysanthemum assembled unigenes and their corresponding homologs in the UniProt database were assigned to Gene Ontology (GO) terms. Biochemical pathway prediction of the chrysanthemum transcripts were annotated and enriched by the Pathway Tools ${ }^{61,62}$.

\section{Quantitative real-time (qRT)-PCR analysis}

Total RNA was extracted from the roots of 40-day-old hydroponic-cultured transgenic and WT chrysanthemum using the RNA plant plus Reagent (Tiangen, Beijing, China).Then, cDNA was synthesized using the PrimeScript first-strand cDNA synthesis kit (TaKaRa, Dalian, China). Each qRT-PCR reaction $(20 \mu \mathrm{l})$ included 1 $\mu \mathrm{l}$ cDNA template, $1 \mu \mathrm{l}$ of both up and down primers $(10 \mu \mathrm{M}), 10 \mu \mathrm{l}$ SYBR Green I, and $7 \mu \mathrm{l}$ RNase-free $\mathrm{H}_{2} \mathrm{O}$. The qRT-PCR assays were carried out according to the StepOne real-time PCR system (Applied Biosystems). All 
reactions were repeated three times, and a chrysanthemum Ubiquitin gene $(\mathrm{CmUBI})$ served as the reference gene $^{60}$. Relative gene transcript abundances were computed with the $2^{-\Delta \Delta C t}$ method $^{56}$. The primers used for qPCR reactions are referred to in Supplementary Table 4.

\section{The cloning and analysis of the promoters}

Genomic DNA extracted from the leaves of "Jinba" using the Plant Whole-genome Extraction Kit (Tiangen, Beijing, China) served as the PCR template. The promoters of four auxin-responsive genes $p C m P I N 2$, $p C m G H 3.1, p C m T A A 1$, and $p C m A B 37 G$ were cloned according to the instructions of Genome-walking Kit (TaKaRa, Dalian, China). A length of $1994 \mathrm{bp} p$ CmPIN2 was a twice genome-walking result. The specific primers for cloning the promoters were designed according to cDNA sequences searched in our RNA-Seq result. Related primers are listed in Supplementary Table 4. PLACE cisacting regulatory DNA elements analysis was completed on the following website (http://bioinformatics.psb.ugent. be/webtools/plantcare/html/).

\section{The expression and purification of CmANR1-His fusion protein}

The open reading frame (ORF) of CmANR1 was cloned from the cDNA of CmANR1 using the paired primers with BamHI and XhoI sites (Supplementary Table 4), and then was constructed into the pET-32a vector, which had a histone (His) tag sequence. Then, the recombinant plasmid was introduced into Escherichia coli BL21 (DE3). The CmANR1 (His)-BL21 bacteria was incubated at $37^{\circ} \mathrm{C}$ constant temperature shaker $(200 \mathrm{rpm})$ for about $2 \mathrm{~h}$. Subsequently the bacteria were treated with $3 \mathrm{mM}$ isopropyl $\beta$-D-1-thiogalactopyranoside (IPTG) for inducing the generation of the CmANR1-His fusion protein. After $4{ }^{\circ} \mathrm{C}$ centrifugation of the bacteria, the precipitate was denatured and renatured with a series of specific concentrations of urea solution $^{63}$. The final fusion protein was transferred to a cobalt chelate affinity resin, which contained the immobilized His-tag. The tube was incubated at $4{ }^{\circ} \mathrm{C}$ for $2 \mathrm{~h}$ on the shaker. After three times separation and abstersion, the protein was collected and detected by western blot using His antibodies (Abcam, Cambridge, UK).

\section{ChIP-qPCR and EMSA analysis}

The chromatin immunoprecipitation quantitative PCR (ChIP-qPCR) tests were performed using the EpiTect ChIP OneDay kit (QIAGEN,Shanghai, China) as described in the previous study ${ }^{63}$. The primers used for ChIP-qPCR are described in Supplementary Table 4. Gel electrophoresis mobility shift assay (EMSA) was carried out following the instructions of the manufacturer in the Light Shift Chemiluminescent EMSA Kit (Thermo, Waltham, MA, USA). Concisely, the biotin-labeled probe was incubated in the $1 \times$
gel/DNA shift binding buffer containing $5 \mathrm{mM} \mathrm{MgCl}_{2}$, $50 \mathrm{mM} \mathrm{KCl}, 2.5 \%$ glycerol, and $10 \mathrm{mM}$ EDTA with or without CmANR1 protein at $24{ }^{\circ} \mathrm{C}$ for $25-30 \mathrm{~min}$. The unlabeled probe with specified concentrations $(50 \times, 100 \times)$ was used for cold probe competition. Related primers are referred to in Supplementary Table 4.

\section{In vivo firefly luciferase (Luc) imaging assay}

The Luc imaging assays were carried out in Nicotiana benthamiana leaves, while the transient expression was performed as previously described ${ }^{64}$. The promoter of CmPIN2 was cloned into pGreenII 0800-LUC vector, generating the reporter $C m P I N 2_{\text {pro }}:$ LUC. The effector $\left(35 S_{\text {pro }}:\right.$ : CmANR1) was constructed by cloning the fragment of CmANR1 (ORF) into the pGreenII 62-SK vector. Then, the recombinant vectors CmPIN2 pro::LUC and $35 S_{\text {pro: }}$ CmANR1 as well as the empty vectors pGreenII 0800-LUC (LUC) and pGreenII 62-SK (35S) were introduced into Agrobacterium strain LBA4404, respectively. The four independent Agrobacterium bacteria with similar $\mathrm{OD}_{600}$ absorbance were 1:1 pairwise mixed. The four kinds of mixed bacteria were infiltrated on the four sites of a same mature N. benthamiana leaf, respectively. A fluorescence imaging instrument (NightOWL II LB983) in conjunction with the Indigo software was used for LUC imaging and luminescence intensity quantification. Infiltrated leaves were sprayed with little luciferin $(100 \mathrm{mM})$, then were put in darkness for $5-10 \mathrm{~min}$ before LUC imaging ${ }^{65}$. Related primers are listed in Supplementary Table 4.

\section{Determination of total IAA in roots}

About $0.2 \mathrm{~g}$ (fresh weight) root samples were prepared and vacuum-dried at $-35^{\circ} \mathrm{C}$ for about $12 \mathrm{~h}$. After quick grind in liquid nitrogen, the powder of the samples was extracted in accordance with the method described by Lin et al. $^{66}$. The total free IAA was detected by the highperformance liquid chromatography.

\section{Statistical analysis}

All samples were analyzed in at least triplicate repeats and represented as the mean \pm standard deviation unless specifically labeled. Significance analysis was determined by Student's $t$-test. $P \leq 0.001$ meant a extremely significant difference, $p \leq 0.01$ represented a significant difference, while n.s. meant no significance.

\section{Results}

\section{CmANR1 promotes AR development in chrysanthemum} under tissue culture conditions

The 35S::CmANR1-1258 (GFP) recombinant plasmid was introduced into chrysanthemum leaf discs using Agrobacterium GV3101-mediated transformation. Subsequently, several positive candidates of CmANR1-transgenic plants, which were preliminarily screened by PCR 
(a)

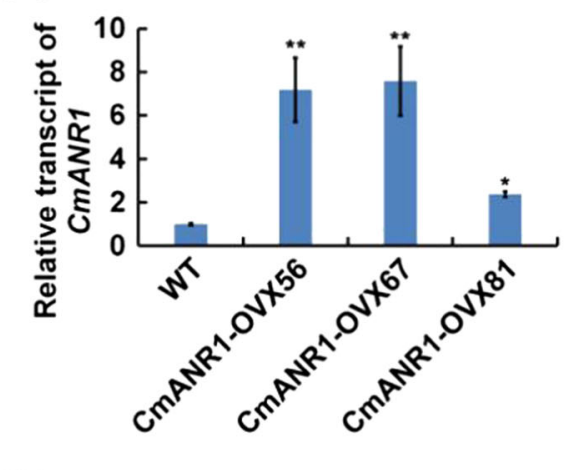

(b)

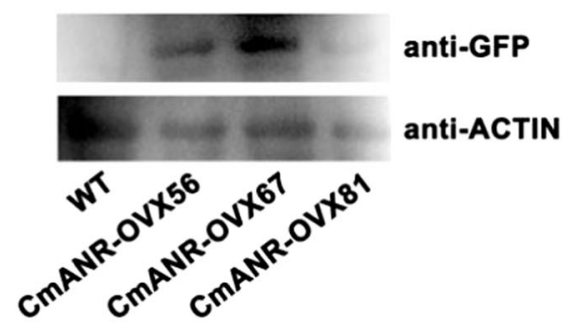

(d)

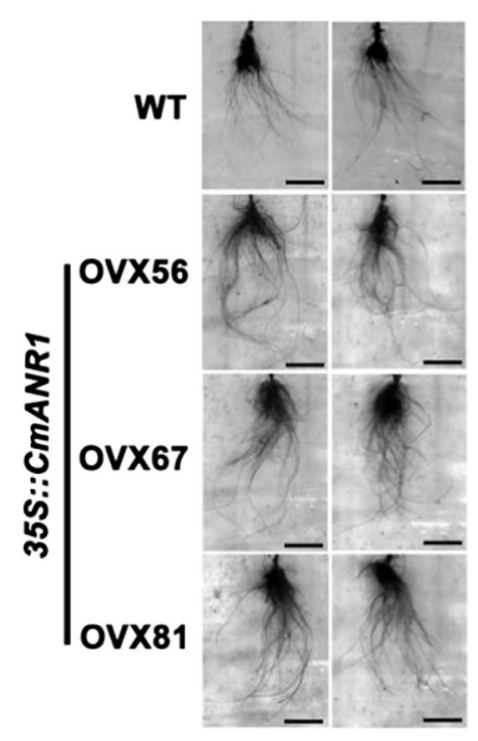

(c) 35S::CmANR1

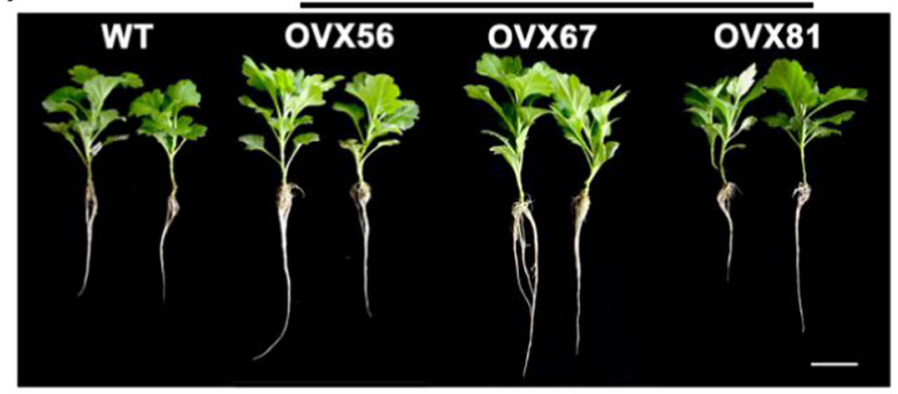

(e)

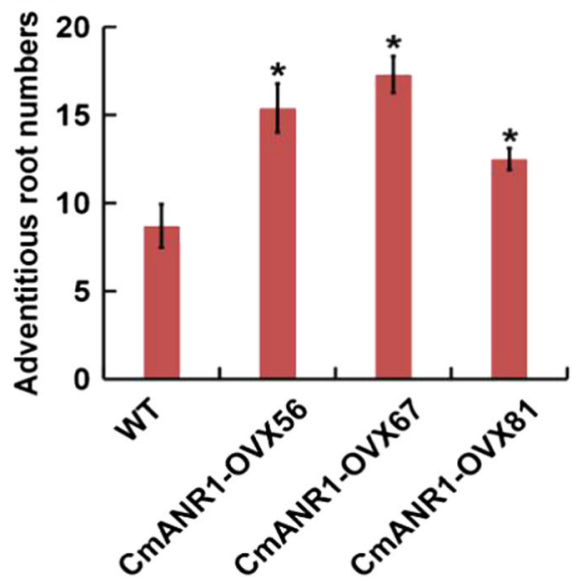

(f)

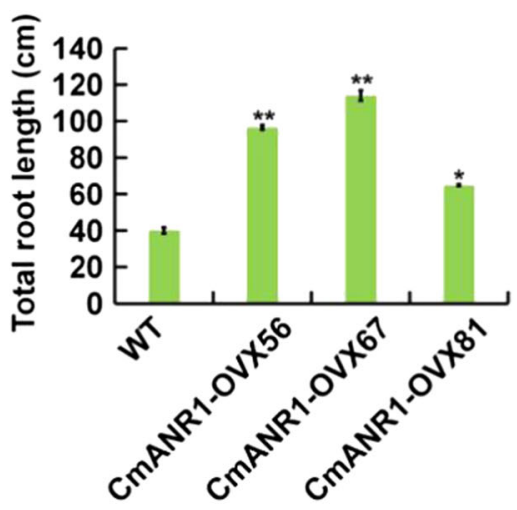

Fig. 1 CmANR1 plays a positive role in adventitious root development in tissue-cultured chrysanthemum. a Relative expression level of the CMANR1 gene in the CmANR1-transgenic and the WT chrysanthemum lines. b The level of the CmANR1-GFP fusion protein in CmANR1-transgenic plants, as determined by immunoblot analysis using an anti-GFP antibody. The anti-actin antibody was used as a loading control. c Phenotypes of CmANR1-OVXs and WT chrysanthemum (tissue culture). Scale bar $=1 \mathrm{~cm}$. $\mathbf{d}$ The root-specific scanning pictures of CmANR1-transgenic and WT plants (tissue culture). Scale bar $=1 \mathrm{~cm}$. e AR number and total root length (f) of CmANR1-transgenic and WT plants (tissue culture). Data are shown as the mean \pm standard error (SE) based on three replicate. Statistical significance was determined using Student's t-test. No significance (n.s.): $p>0.01 ;{ }^{*} p<0.01 ;{ }^{* *} p<0.001$ 
detection using the genomic DNA as the template, were further identified by qRT-PCR analysis (Supplementary Fig. S1). Among them, three independent CmANR1transgenic lines (CmANR1-OVX56, -OVX67, -OVX81) with significantly distinct expression levels of CmANR1 were selected for further investigation (Fig. 1a). Immunoblotting assays showed that the CmANR1 protein accumulated in these three CmANR1-transgenic chrysanthemums much more than in the WT control (Fig. 1b). These three CmANR1-transgenic and WT chrysanthemums were then rooted and cultivated in vitro. Following this, the CmANR1-overexpressing plants exhibited a stimulation on AR development compared with the WT plants (Fig. 1c, d). Remarkably, the numbers and total lengths of the ARs in the CmANR1-OVXs lines were increased by $43.7-98.8 \%$ and $61.8-184.5 \%$, respectively, in comparison to the WT plants (Fig. 1e, f). Therefore, a significant increase in both root surface and volume was showed in the transgenic plants compared to the WT plants (Supplementary Table 1). These results suggest a positive role of CmANR1 on AR development in chrysanthemum.

Surprisingly, fewer LRs were found in both the CmANR1-transgenic and WT plants under tissue culture conditions, which seemed to be in contrast to the positive role of ANR1 on LR growth in Arabidopsis ${ }^{51,56}$. To further confirm the role of CmANR1 on AR development, AR rooting experiments were performed using CmANR1overexpressing (CmANR1-OE3, OE6 and OE9) and WT Arabidopsis seedlings, which were obtained in our previous study ${ }^{56}$. Only the hypocotyls of those plants were placed on the MS medium and the MS medium with 0.1 $\mu \mathrm{M}$ IBA added, and cultivated vertically under dark conditions. Following this, the seedlings developed new ARs after about 1 week of growth. The total length and numbers of ARs in the CmANR1-transgenic seedlings were significantly increased compared to the WT plants, with respective increases of $8.8-56.6 \%$ and $50.4-150.2 \%$ (Supplementary Fig. S2). Additionally, the exogenous application of IBA almost abolished the developmental differences of the ARs between the transgenic and WT seedlings, suggesting that auxin may have some relationship with AR development.

\section{CmANR1 promotes AR and LR development in chrysanthemum under hydroponic culture conditions}

To better evaluate the function of CmANR1 in root system development, the WT and CmANR1-overexpressing chrysanthemums exhibiting uniform growth under tissue-cultured conditions were then cultivated hydroponically. The results showed that the CmANR1overexpressing chrysanthemums possessed a much more extensive root system, including more ARs and LRs, compared to the WT plants after about 35-40 days of growth (Fig. 2a, b). CmANR1-overexpressing chrysanthemum exhibited a significant increase in root volume and total root length compared to the WT plants, by $0.6-1.9$-fold and $0.5-1.2$-fold, respectively (Fig. 2c, d). Meanwhile, the numbers of ARs and LRs in the three CmANR1-overexpressing plants were much greater than those of the WT plants, with 0.3-0.5-fold and 0.2-1.1fold increases, respectively (Fig. 2e, f). The stronger root system of the CmANR1-transgenic plants indicated the positive effect of CmANR1 on rooting in chrysanthemum under hydroponic conditions. Furthermore, a significant stimulation on shoot height was observed in the CmANR1-overexpressing chrysanthemums compared to the WT plants, elevated by 16.1-51.9\% (Supplementary Table 2). The increase in shoot height of the CmANR1$O V X s$ plants may be attributed to a feedback-enhanced uptake of water and nutrients by the more extensive root system.

\section{Transcriptome sequencing of CmANR1-overexpressing and WT chrysanthemum roots}

To reveal the underlying mechanism of CmANR1 in controlling root system development, strand-specific RNA sequencing libraries from the roots of the WT and CmANR1-overexpressing chrysanthemums were constructed. De novo assembly of the valid cleaned reads produced 51,481 unigenes with a mean length of $653 \mathrm{bp}$ and a longest length of $8281 \mathrm{bp}$. The length distribution of the assembled unigenes is exhibited in Supplementary Table 3. Then, we annotated the assembled unique transcripts by BLAST against several protein databases. A total of 24,292 (47.2\%), 36,870 (71.7\%), 37,115 (72.2\%), 28,442 (55.3\%), 30,328 (59.0\%), 30,392 (59.1\%), and 12,050 (23.4\%) unique transcripts obtained significant hits (identity $\geq 30 \%, E$-value $\leq 1 \mathrm{e}-5$ ) in the Swiss-Prot, TrEMBL, GenBank NR, Pfam, eukaryotic orthologous groups (KOG), GO (http://www.geneontology.org/), and Kyoto Encyclopedia of Genes and Genomes (KEGG) databases, respectively. Remarkably, "Signal transduction mechanisms" was the second-most abundant group in these KOG functional categories after "Posttranslational modification, protein turnover, chaperones", irrespective of the poorly characterized ones (Supplementary Fig. S3a). In contrast, the GO terms "metabolic process" in the biological process category, "cell" in the cellular category, and "binding" in the molecular function category were the most enriched in these three categories, respectively (Supplementary Fig. S3b). In the KEGG pathway classification, "Carbohydrate metabolism" in the metabolism category was the most abundant group (Supplementary Fig. S3c).

Subsequently, FPKM (Fragments Per Kilobase of transcript per Million fragments mapped) was used to evaluate the expressional abundances of the assembled 
(a)

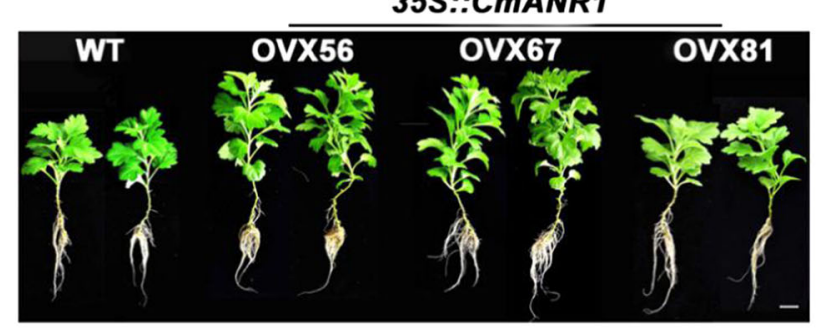

(b)

(c)
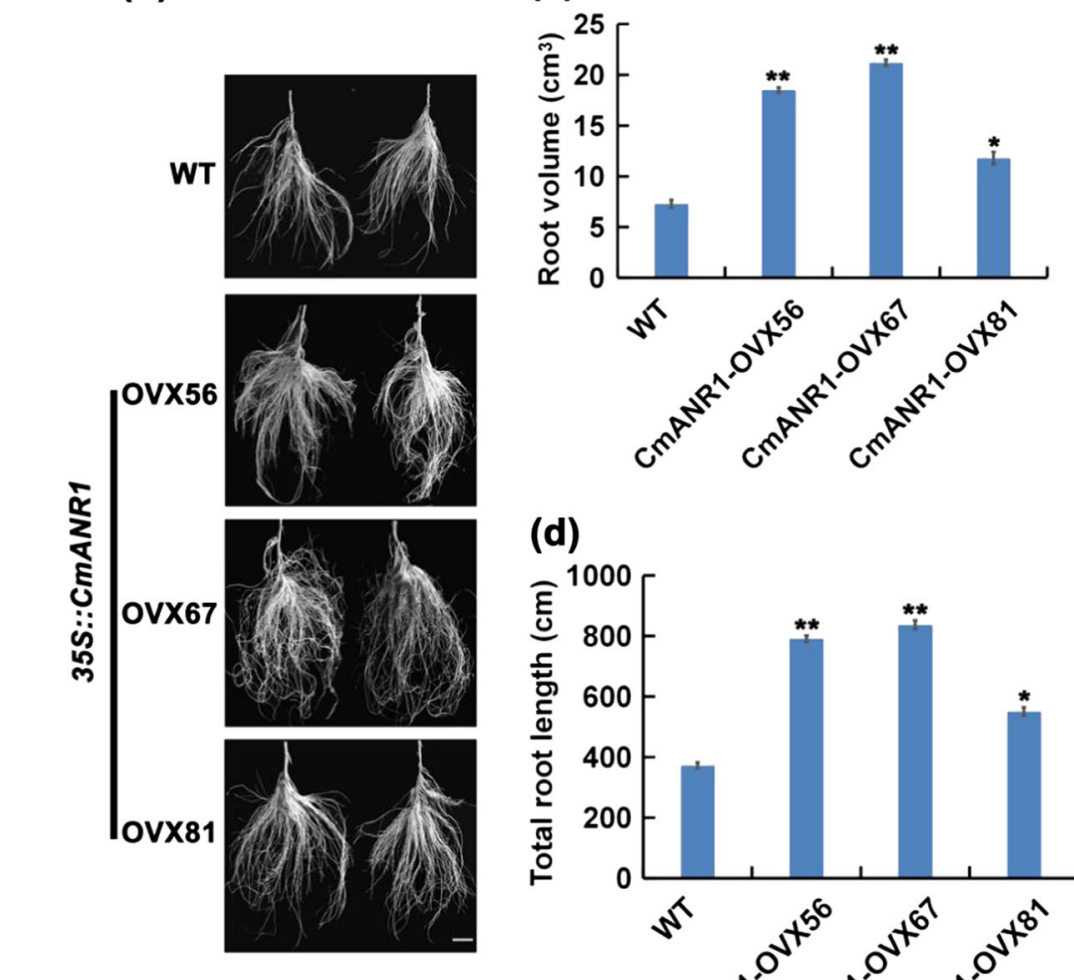

(d)

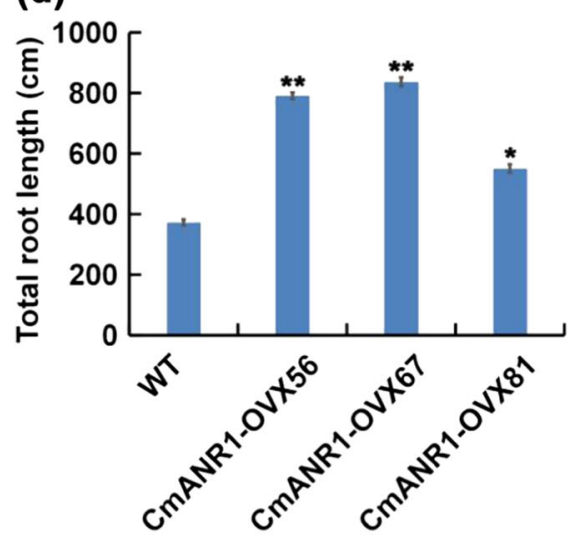

(e)

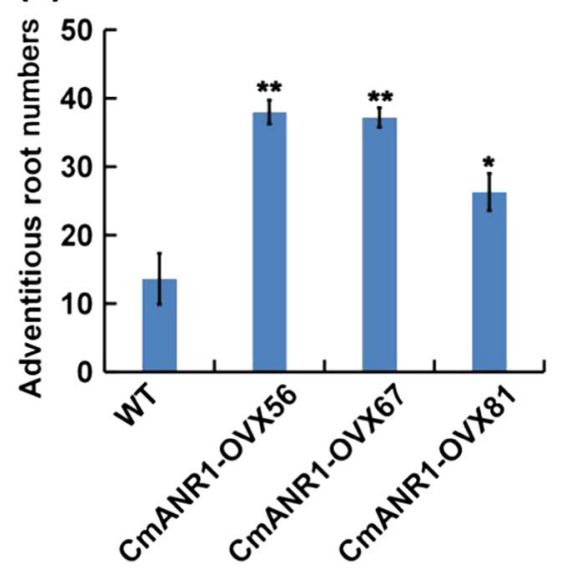

(f)

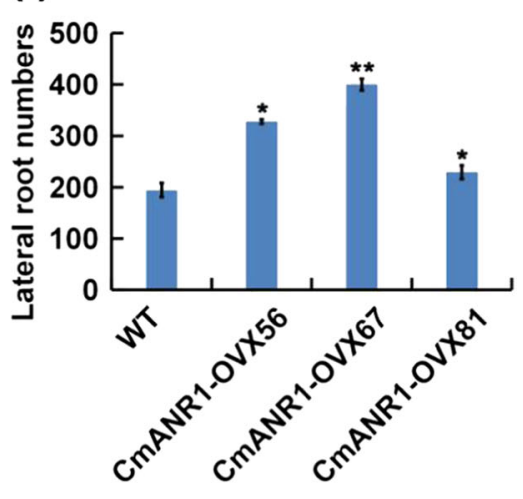

Fig. 2 CmANR1 promotes both adventitious root and lateral root development in hydroponic-cultured chrysanthemum. a Phenotypes of CmANR1-transgenic and WT chrysanthemums under hydroponic culture conditions. Scale bar $=1 \mathrm{~cm}$. b The root-specific magnified pictures of CmANR1OVXs and WT plants (hydroponic culture). Scale bar $=1 \mathrm{~cm}$. $\mathbf{c}-\mathbf{f}$ The root volume (c), total root length (d), AR number (e), and LR number (f) of CmANR1transgenic and WT plants. The data represent the means \pm SE of three independent experiments. Statistical significance was determined using Student's 
(a)

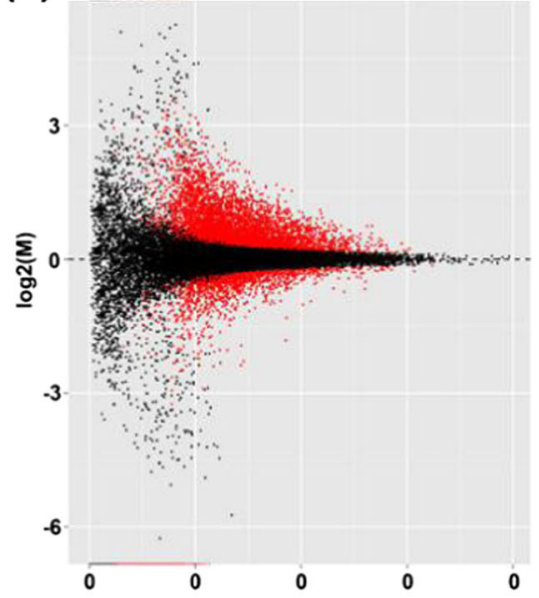

(b)

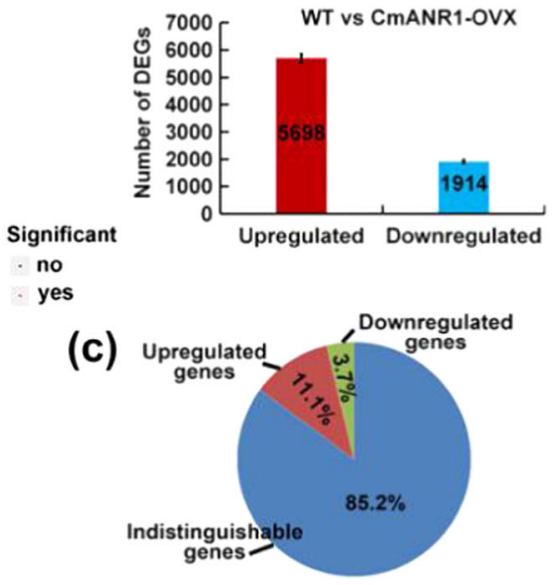

(d)
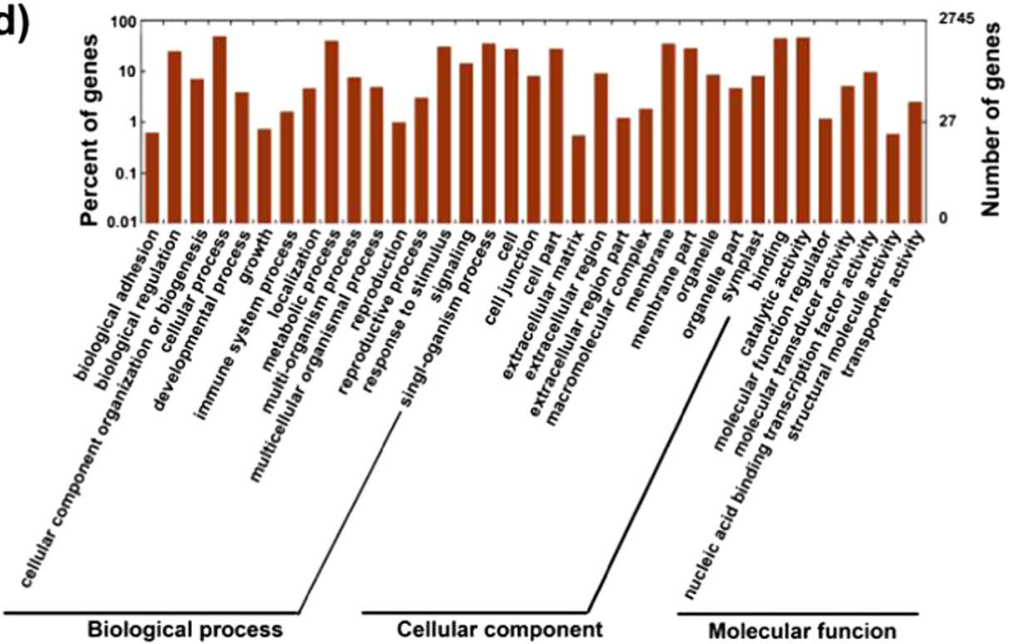

(e)

Pathway enrichment

Zeatin biosynthesis Sulfur metablism Stilbenoid, diarylheptanoid and gingerol biosynthesis Starch and suerose motabolism Plant-pathogen interaction Plant hormone signal transduction Phenylpropanoid biosynthesis Pentose and glucuronate interconversion Nicotinate and nicotinamide metabolism Linoleic acid metabolism Inositol phosphate metabolism Glycerophospholipid metabolism Flavonoid biosynthesis Ether lipid metabolism Endocytosis Cysteine and methionine metabolis Carotenoid biosynthesis Biosynthesis of unsaturated fatty acids Ascorbate and aldarate metabolism Amino sugar and nucleotide sugar metabolism alpha-Linolenic acid metabolism
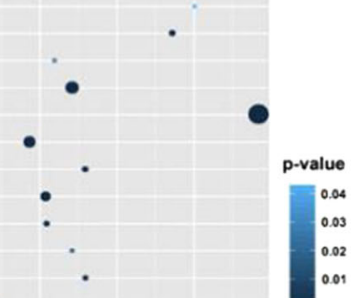

Gene_number

25

. 75

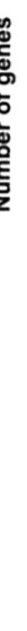


Fig. 3 Annotation enrichment of DEGs in the roots of CmANR1-OVXs in contrast with WT chrysanthemum. a The volcano plot of the DEGs. A small dot in the picture represents a unigene; the $x$-axis represents the $\log _{2}($ fold change) of a gene expression difference between WT and CmANR1OVXs; the $y$-axis represents the negative log Kow of the $p$ value of the gene expression change. Unigenes with significant differential expression are indicated with a red dot. $\mathbf{b}$ The numbers of up-regulated and down-regulated unigenes in the RNA-Seq results. c Pie chart representing the percentage of up- and down-regulated unigenes and indistinguishable unigenes in RNA-Seq data. $\mathbf{d}$ GO enrichment analysis of the DEGs. The $x$ coordinate is the GO classification, which is the secondary function of GO; the left vertical coordinate is the percentage of the DEGs; and the right shows the corresponding numbers. e KEGG bubble chart of the DEGs. The bubble size represents the number of DEGs, and the bubble color represents the $p$ value. The rich-factor equals the number of DEGs/the number of background genes in a certain signaling pathway

unigenes based on the transcriptome sequencing data. The volcano plot showed the relationship between the significance of the $p$ value and fold change of all the differentially expressed unigenes (DEGs) (Fig. 3a). Simultaneously, the MA Value Plot presented the distribution and differences in DEGs in the WT and CmANR1-overexpressing chrysanthemums (Supplementary Fig. S4a). In addition, a heatmap of the DEGs provided a visual illustration of the expressional differences between the WT and CmANR1-overexpressing chrysanthemums, with high expression levels shown in red and low expression levels shown in green (Supplementary Fig. S4b). A total of 7612 DEGs were identified in the CmANR1-overexpressing chrysanthemums compared to the WT plants (Fig. 3b; Supplementary Appendix S1). Among them, 5698 (11.1\%) were up-regulated and 1914 (3.7\%) were down-regulated (Fig. 3b, c; Supplementary Appendix S1). Subsequently, these up-regulated and down-regulated genes that were involved in the main biological functions, as well as the biochemical metabolic and signal transduction pathways in GO and KEGG enrichment, were analyzed (Supplementary Appendix S2 and S3). GO terms involving "catalytic activity" and "binding" in the molecular function category and "metabolic process", "response to stimulus", and "signaling" in the biological process category were highly abundant in the GO enrichment annotation of the up-regulated unigenes (Fig. 3d). Meanwhile, "Plant hormone signal transduction" was abundant in the KEGGenrich analysis and enriched bubble diagram of the upregulated unigenes (Fig. 3e). These information suggested that CmANR1 might participate in the regulation of plant hormone signaling processes. However, DEGs being classified to "Plant-pathogen interaction" term in KEGGenrich analysis accounted for the most abundant, which attracted our attention and would be discussed later.

\section{Expressional profiling of the genes involved in root system development in chrysanthemum}

Four groups of potential candidate unigenes from the DEGs associated with root system development, including auxin-responsive group, calcium $\left(\mathrm{Ca}^{2+}\right)$ signaling-related group, ethylene-related group, and cell cycle group, were selected for further investigation. The corresponding ID numbers and $\log _{2}$ (fold changes) values of these unigenes are listed in a supplementary file (Supplementary
Appendix S4). The heatmaps display the average absolute expression values after $\log _{2}$ transformation between WT and CmANR1-overexpressing chrysanthemums (Fig. 4). Among them, the expressions of some auxin transport unigenes (PIN2, AUX1, AB37G, and $A B 11 B$ ) and a range of auxin-responsive protein genes (SAU20, SAU36, $R H M 1$, and $X T H 20$ ) showed a significant increase in the CmANR1-overexpressing chrysanthemum compared to the WT plants in the auxin-responsive group (Fig. 4a). $\mathrm{Ca}^{2+}$ signaling-related unigenes, such as the calciumbinding protein genes CMLs (CML45, CML23, and $C M L 50)$ and calcium-dependent protein kinase genes CDPKs (CDPK6, CDPK10, CDPK30, and CDPK33), were also found to be significantly up-regulated in the CmANR1-overexpressing chrysanthemums compared to the WT plants (Fig. 4b). The ethylene biosynthesis genes $A C S 7$ and ethylene-responsive TF genes ERFs (ERF3, $E R F 78$, and ERF109) were significantly up-regulated in the CmANR1-overexpressing chrysanthemums compared with the WT plants (Fig. 4c). In addition, the expressions of cell cycle-related genes (CYCB1-4, CYCD3-2, ALISs, $M L H 1$, and $M K K 4)$ were also significantly altered in the CmANR1-overexpressing chrysanthemums compared to the WT plants (Fig. 4d).

To further validate our RNA-Seq results, 18 unigenes that were marked in red in Fig. 4 from the four groups were selected for qRT-PCR verification. As a result, most of these unigenes were significantly increased in the CmANR1-overexpressing chrysanthemums compared to the WT plants (Fig. 5a, b). Additionally, we found that the exact fold changes of the detected unigenes varied between the qPCR analysis and RNA-Seq expression data (Fig. 5c), but the high correlation $\left(R^{2}=0.882\right)$ correlated with a simple linear regression equation $y=0.96 x+0.467$ demonstrated that a good consistency existed between the two experimental methods (Fig. 5d).

\section{CmANR1 facilitates auxin polar transport by direct transcriptional activation of CmPIN2}

Accumulating evidence suggests that auxin plays a central role in both AR and LR development ${ }^{30}$. To determine the causality between auxin and root system development, we measured the endogenous free IAA content in the roots of the WT and CmANR1-transgenic plants. As observed with the up-regulated expressions of 


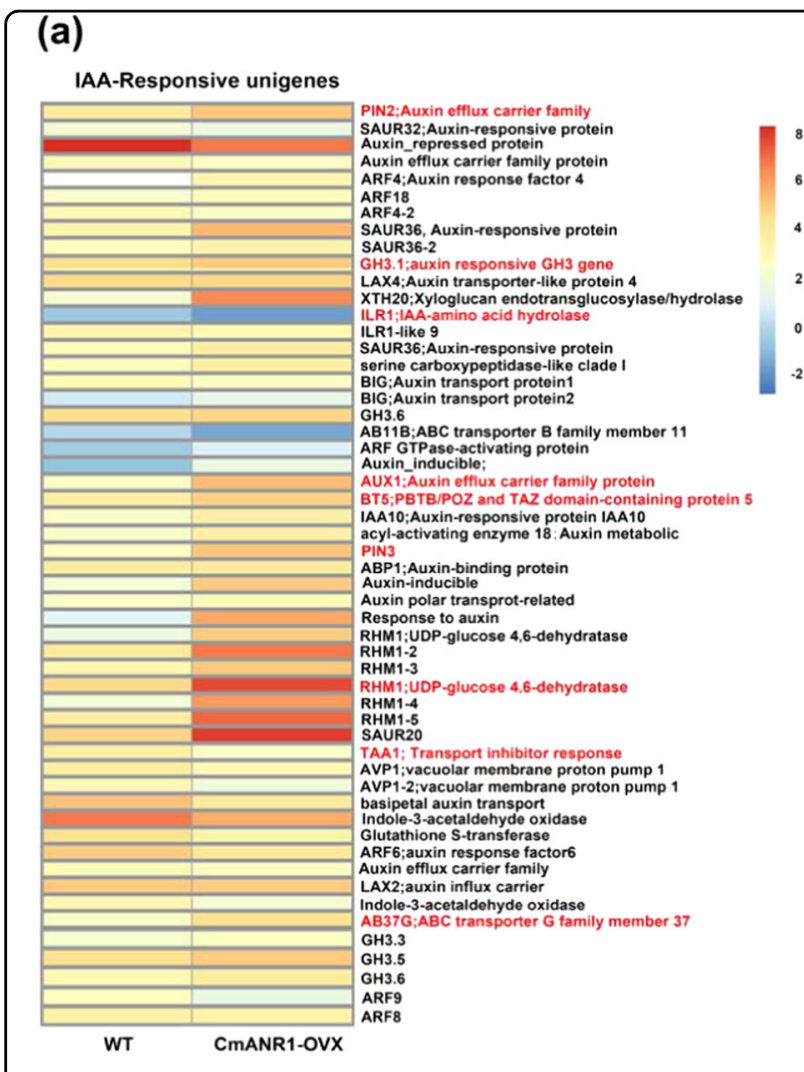

(b)
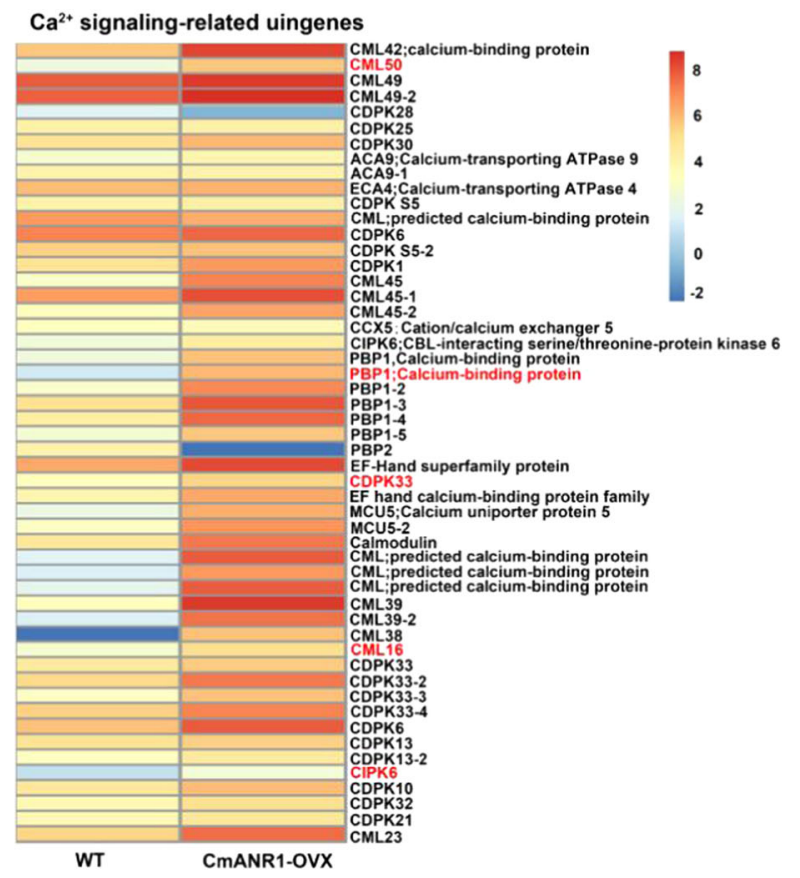

(d)

(c)

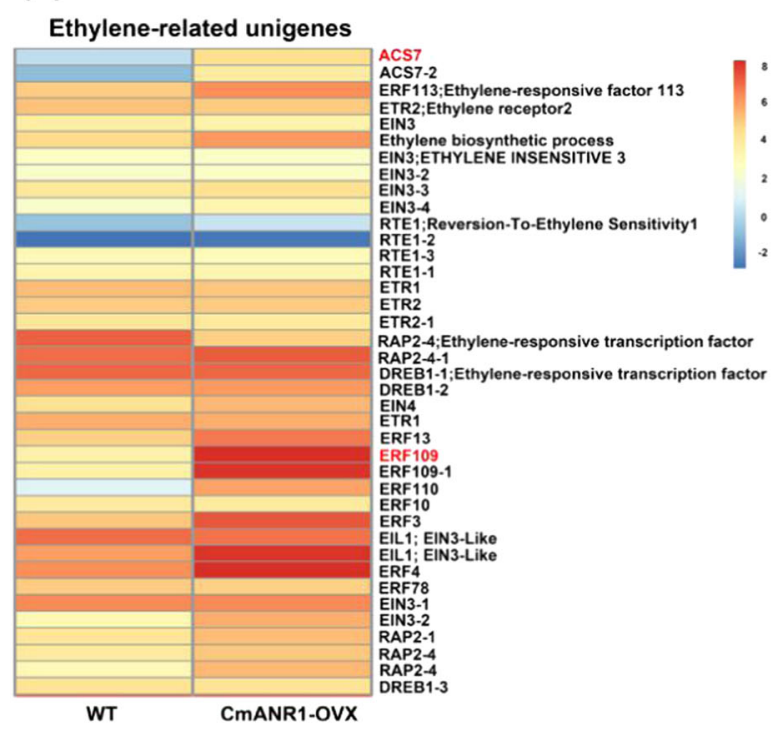

Cell cycle-related unigenes

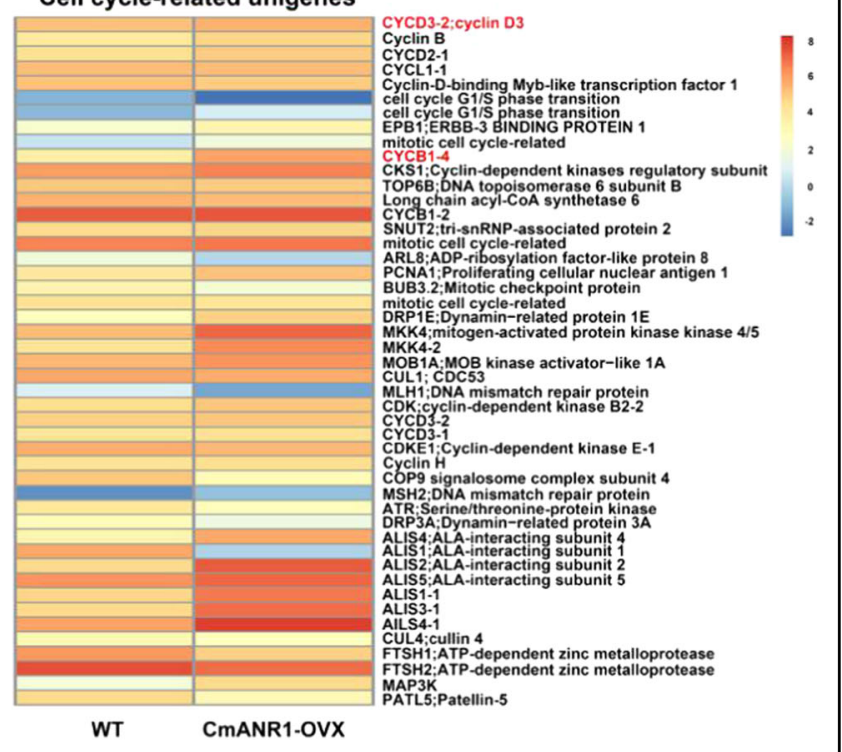

Fig. 4 Heatmaps of four groups of candidate unigenes associated with root system development in the DEGs. a Heatmap of auxinresponsive unigenes. $\mathbf{b}$ Heatmap of $\mathrm{Ca}^{2+}$ signaling-related unigenes. $\mathbf{c}$ Heatmap of ethylene-related unigenes. $\mathbf{d}$ Heatmap of cell cycle-related unigenes. Differences in expression levels are represented by color gradients. Red and orange strips indicate highly to moderately up-regulated unigenes, while dark blue to light blue strips represent highly to moderately down-regulated unigenes. The ID numbers of the four groups of unigenes are provided in Supplementary Appendix S4. The red unigenes in the four groups were selected for qRT-PCR

auxin polar genes, such as PIN2, PIN3, and AUX1 (Fig. 5a), the free IAA level in the roots of CmANR1transgenic chrysanthemum was elevated by $23.4-89.4 \%$ compared to the WT plants (Fig. 6a), suggesting that auxin was the underlying cause of the more robust root system in the CmANR1-transgenic plants. 


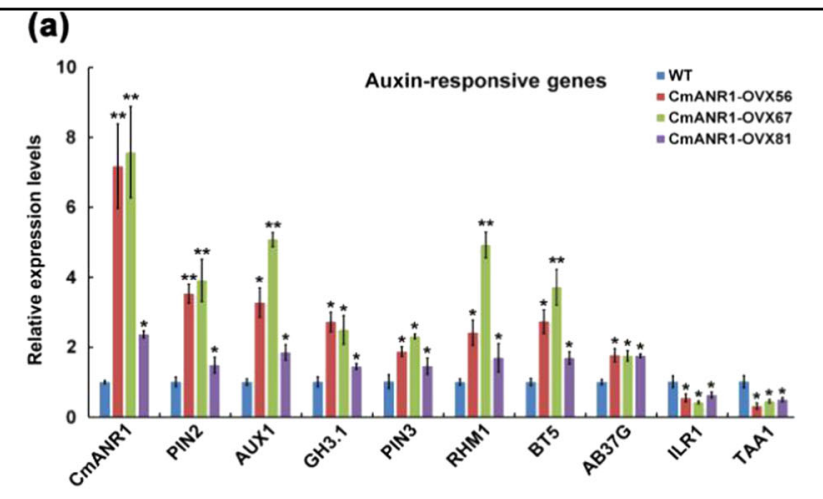

(c)

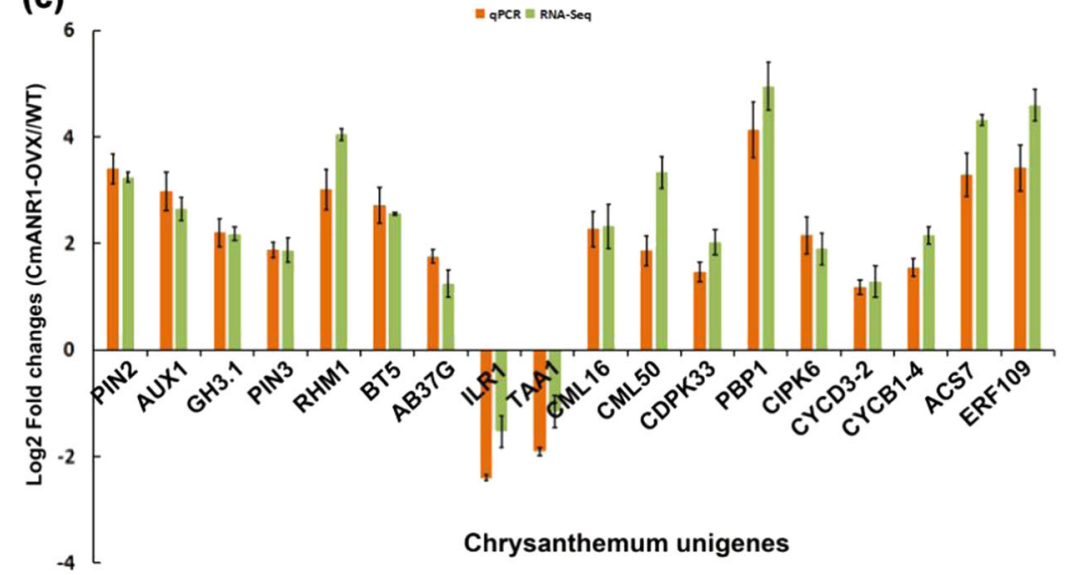

(b)

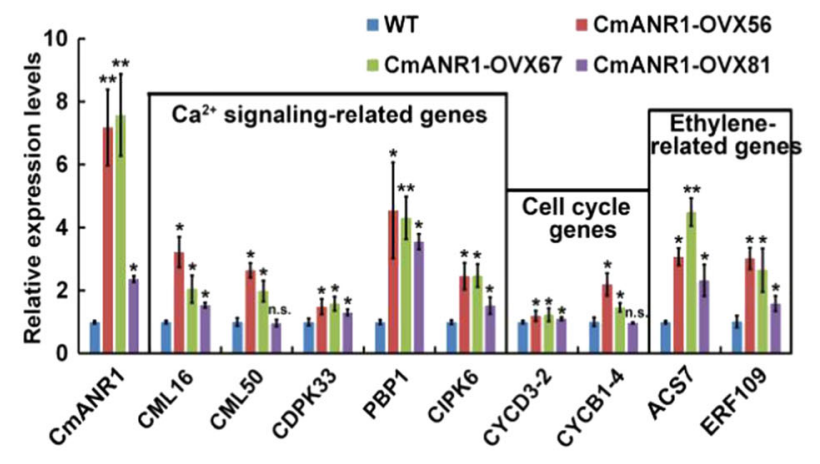

(d)

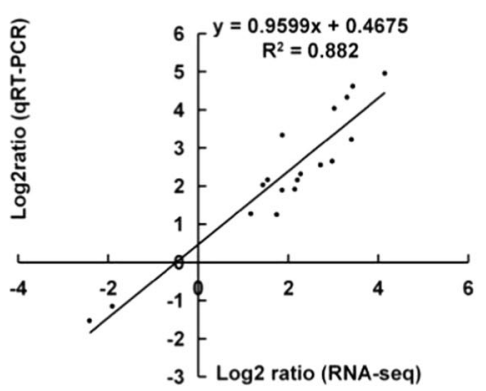

Fig. 5 Verification of the RNA-Seq results by qRT-PCR. a The relative transcript abundance of some IAA-responsive unigenes that were selected based on their differential expression in the roots of WT vs. CmANR1-transgenetic plants. $\mathbf{b}$ The relative expression level of the unigenes in Ca ${ }^{2+}$ signaling-related, ethylene-related, and cell cycle-related groups in the roots of WT and CmANR1-transgenetic plants. c Comparison of the expression level of unigenes between the RNA-Seq and qRT-PCR. $\mathbf{d}$ Scatter diagram of the log ratios ( $\log _{2}$ FC) of the unigenes. The qRT-PCR data were normalized to the internal control CmUBI. Note that in $\mathbf{a}$ and $\mathbf{b}$, data are shown as the mean \pm SE based on three or more replicate. Statistical significance was determined using Student's t-test. n.s.: $p>0.01 ;{ }^{*} p<0.01 ;{ }^{* *} p<0.001$

MADS-box TFs bind to specific DNA sequences with an overall consensus of $\mathrm{CC}(\mathrm{A} / \mathrm{T})_{6} \mathrm{GG}$, called CArG-box motifs ${ }^{67}$. To further verify whether CmANR1 directly activates the expression of auxin-responsive genes, the promoters of CmPIN2, CmGH3.1, CmTAA1, and $C m A B 37 G$ were cloned by the Genome-Walking method. Plant cis-acting regulatory DNA elements (PLACE) analysis found that there was one CArG-box motif in the promoters (Supplementary Fig. S5). Subsequently, the ChIP-qPCR assays were carried out using CmANR1transgenic and WT chrysanthemum. The results demonstrated that the CArG-box motif of CmPIN2 was significantly recruited by CmANR1, while others were not (Fig. 6b). The results provided in vivo evidence for the binding of CmANR1 to the CmPIN2 promoter on the CArG-box motif site.

To further validate the binding of CmANR1 to the CArG-box recognition site in the CmPIN2 promoter in vitro, EMSA analysis was conducted with an oligo- probe containing the CArG-box motif using the purified CmANR1-His fusion protein. The result showed that specific DNA-CmANR1 protein complexes were detected when the CArG-box motif-containing sequence was used as the labeled oligo-probe. When increasing the amounts of the unlabeled competitive probe with the same sequence, we found that the binding complexes were reduced. However, the competition was not existed in the mutated version. The specificity of the competition verified the physical interaction between the CmANR1 protein and CmPIN2 promoter, which required the specific CArG-box cis-element (Fig. 6c).

To examine whether CmANR1 directly activates CmPIN2, an in vivo firefly Luc imaging assay was performed. Constructs containing $35 S_{\text {pro }}:$ CmANR1 (pGreenII 62-SK) and the CmPIN2 pro::Luc (pGreenII 0800-Luc), as well as Luc (pGreenII 0800-Luc) and 35S pro (pGreenII 62-SK) were respectively co-infiltrated into tobacco leaves to express these fusion proteins transiently. The results 

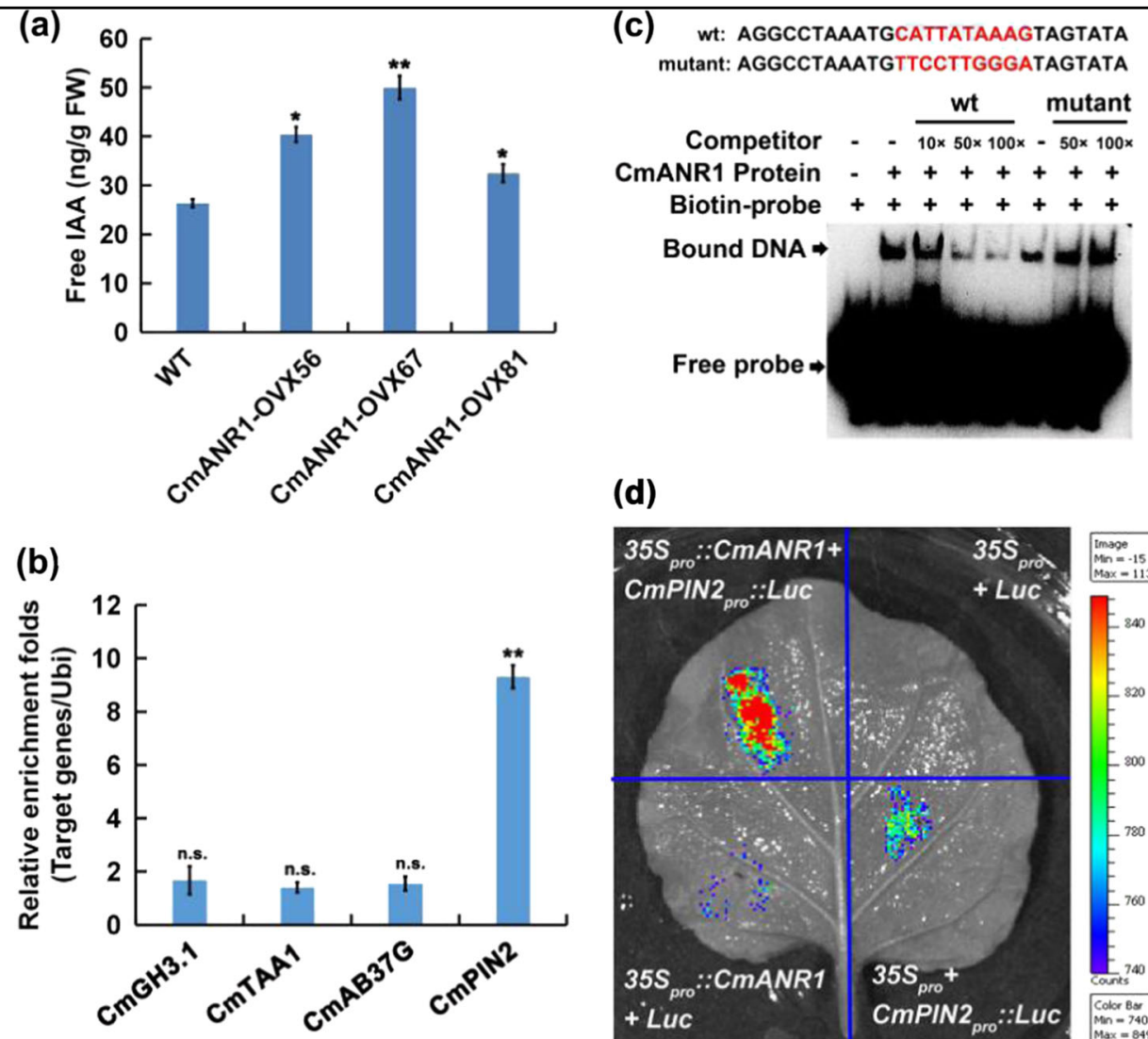

(d)

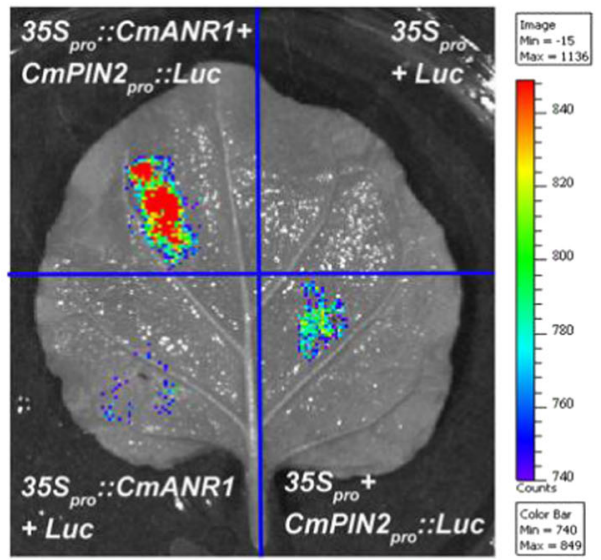

Fig. 6 CmANR1 binds directly to the CmPIN2 promoter. a Total free IAA content in the roots of 20-day-old WT and CmANR1-transgenic chrysanthemums. $\mathbf{b}$ The relative enrichment folds of the fragment containing the CArG-box motif by ChIP-qPCR in the promoters of four auxinresponsive unigenes. c CmANR1 directly binds to the CArG motif presented in pCmPIN2 in vitro, as indicated by EMSA. Competition for CmANR1 binding was performed with 10x, 50x, and 100x unlabeled probes (wt) or 50x and 100x CArG-box-mutated probes (mut). The symbol " + " indicates presence and "-" indicates absence. $\mathbf{d}$ The Luc imaging assay indicates that CmANR1 activates the expression of CmPIN2. Representative images of Nicotiana benthamiana leaves $72 \mathrm{~h}$ after infiltration are shown. Statistical significance was determined using Student's $t$-test. n.s.: $p>0.01 ;{ }^{*} p<0.01$; ${ }^{* *} p<0.001$

demonstrated that a strong luminescence signal was

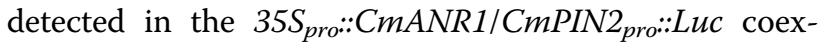
pression region, but no or very weak luminescence signal was detected in the negative controls (Fig. 6d). These results indicate that CmANR1 directly activates CmPIN2 transcription.

Taken together, our results suggest that CmANR1 activates the transcription of the CmPIN2 gene by direct binding to the CArG-box motif in its promoter.

\section{Discussion}

MADS-box TF genes have been extensively identified as the important regulators of flowering time, floral organ identity, and flower development ${ }^{68-72}$. In contrast, fewer MADS-box TF genes have been reported to regulate root development ${ }^{27,55,56}$. With regards to the molecular regulatory mechanisms on root development, MADS-box TF genes are seldom an immediate regulator. In fact, these MADS-box genes indispensably exert their effects on root development through cross-talks with other signals, such as the cross-talk between OsMADS25 and the nitrate signal $^{54}$, and GmNMHC5 with the sucrose signal in controlling root development ${ }^{55}$. Remarkably, the functional mechanism of a large part of MADS-box genes on root development is mainly auxin dependent, such as ANR1, AGL21, and XAL2/AGL14 ${ }^{27,52,53}$. Interestingly, our previous study on CmANR1 demonstrated that a nitrate signaling pathway as well as auxin-related processes interacted under the integration of the CMANR1 gene, giving rise to the proliferation of LR growth in Arabidopsis $^{56}$.

A great deal of evidence suggests that auxin is central to both LR development ${ }^{9,13}$ and AR formation ${ }^{11,12}$. In this study, the free IAA content was highly elevated in the roots of CmANR1-OVXs plants compared to the WT control (Fig. 6a), which provided a reasonable explanation for the better developed ARs and LRs in CmANR1transgenic chrysanthemums. It is well known that PINs 


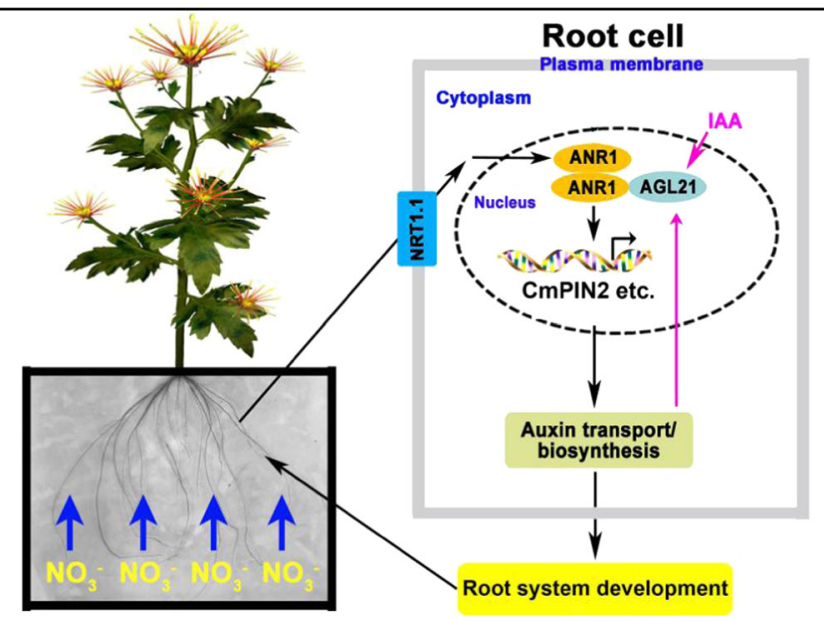

Fig. 7 The working model of CmANR1 in root development in chrysanthemum: CmANR1 promotes both AR and LR development by transcriptional activation of CMPIN2 in the roots

and $A U X / L A X s$ are two dominating groups of auxin efflux/influx carrier genes that are essential for auxin polar transport in plants $^{32}$. Moreover, PIN genes have been reported to provide rate-limiting functions in auxin movement ${ }^{29,34}$. In our study, the expressions of the auxin transport genes PIN2, PIN3, and AUX1 were significantly up-regulated in the roots of the CmANR1-transgenic plants compared to the WT control (Fig. 5a). Although auxin is also synthesized in the root tips, the several included auxin synthesis unigenes (e.g., YUC1, AAO-Like, AAO2, NIT2-Like, and NIT4) in the RNA-Seq results showed no obvious expressional differences in the roots between WT and CmANR1-OVXs. Given the role of polarity in auxin transport, the increased auxin in the roots of the CmANR1-transgenic plants was more likely due to shoot-to-root auxin transport under the mediation of auxin transport carrier genes, but not as a direct result of auxin biosynthesis in the roots. Furthermore, CmANR1 could directly activate CmPIN2 transcription (Fig. 6), confirming the possibility of root-ward auxin transport at least partially by CmPIN2 in CmANR1-transgenic chrysanthemum.

In addition, calcium, as an important signaling messenger, was found to be related with the regulation of AR or LR development via cross-talks with auxin. $\mathrm{Ca}^{2+}$ and CDPK act as the downstream messengers during auxininduced AR formation in cucumber ${ }^{16}$. AtCIPK6 significantly affects LR formation by positively regulating root basipetal and shoot-to-root auxin transport ${ }^{73}$. OsCBL1 is required for LR development in rice by mediation of auxin biosynthesis ${ }^{17}$. In line with previous studies, a multitude of $\mathrm{Ca}^{2+}$-signaling-related genes, including CML6, CML45, CML50, CIPK6, CDPK10, and $C D P K 33$, as well as $P B P 1$, were significantly up-regulated in the roots of CmANR1-overexpressing chrysanthemum compared to WT plants (Figs. 4b, 5b), which indicated possible cross-talk of calcium signaling with auxin-related physiological processes in regulation of root development under the mediation of CmANR1. Moreover, the complex interaction of auxin with ethylene in root development has been well documented ${ }^{21,22,74}$. Auxin can stimulate ethylene biosynthesis by increasing ACS transcription ${ }^{75}$ and positively regulating the ethylene-mediated inhibition of root growth ${ }^{76}$. Ethylene has a positive role in the regulation of auxin synthesis and promotes basipetal auxin polar transport in the roots, resulting in increased auxin as well as a subsequent triggering of ethylene-mediated root growth inhibition ${ }^{77-79}$. Notably, in a present study, the expression levels of numerous ethylene-related unigenes were markedly varied in the roots of CmANR1transgenic plants compared with the WT plants (Fig. 4c), such as ACS7, ERF13, and ERF109. However, the major components in the ethylene signaling pathway, including ETR1, ETR2, EIN2, EIN3, and EIL1, showed no obvious expressional differences between the WT and CmANR1$O V X$, suggesting the absence of the ethylene signaling pathway in this context. There seems to be a paradox between the ethylene signaling and RNA-Seq results here. A possible explanation is that the ERFs (ERF13 and ERF109) have other functions, or otherwise are not the main determinants involved in the ethylene signaling pathway. In fact, ERF109, which is highly jasmonic acid (JA)-responsive, plays an important role in mediating the connection of JA signaling with auxin biosynthesis during LR formation in Arabidopsis ${ }^{80}$. Therefore, ethylene biosynthesis rather than signaling may take part in regulating root development in chrysanthemum. Further researches are needed to prove this hypothesis.

In our transcriptome sequencing results, thousands of unigenes were significantly differentially expressed in the 
root samples of CmANR1-OVXs compared to the WT control (Fig. 3). The annotations on these DEGs were highly informative. In addition to the anticipated DEGs, there were some unexpected results that required further assessment. For example, the "Plant-pathogen interaction" term accounted for the most abundant group in the KEGG enrichment analysis on the up-regulated DEGs (Fig. 3e). In terms of pathogen defense in plants, the phytohormone JA naturally becomes a primary consideration. As a biotic and abiotic stress-related hormone, JA is essential for immunity and development in plants. In addition, methyl JA was proved to repress root growth in Arabidopsis $^{81}$. Moreover, a previous study on AR formation in Arabidopsis reported that the auxin-inducible Gretchen Hagen3 (GH3) genes, GH3.3, GH3.5, and GH3.6, could lower JA content in the roots by down-regulating JA biosynthesis and enhancing JA conjugation. GH3 genes were found to fine-tune AR formation by a combination of auxin and JA regulatory pathways ${ }^{11}$. Inspired by the above studies, we discovered that the auxin-responsive GH3 unigenes (GH3.1, GH3.5, and GH3.6) were significantly up-regulated in the roots of CmANR1-OVXs plants compared to the WT control. Conversely, three JAamido synthetase (JAR1) unigenes were down-regulated in our RNA-Seq results. Therefore, based on this observation, JA signaling may possibly be involved in AR development by cross-talks with auxin under the integration of CmANR1 in chrysanthemum.

In conclusion, we have provided a summary of a working model of the MADS-box TF gene CmANR1 on the regulation of both AR and LR development in chrysanthemum (Fig. 7). When there was a higher concentration of nitrate in the surviving environment of chrysanthemum roots, NRT1.1, as the nitrate sensor on the root cell plasma membrane, sensed and transferred the external nitrate signal to ANR1 in the nucleus ${ }^{82,83}$. Then, the nuclear-localized $A N R 1$ was rapidly enriched in response to the nitrate signal, forming more ANR1/ANR1 homodimers $^{56}$. Subsequently, the TF gene ANR1 upregulated the expressions of several auxin polar transport genes, such as PINs and AUX1, thereby facilitating rootward auxin transport. Alternatively, in our previous study, local auxin biosynthesis was elevated concurrently ${ }^{56}$. The resulting increased auxin in the roots promoted both AR and LR development in chrysanthemum. In contrast, the increased auxin in the roots may feedback-regulate the expression of a member of the same clade, $A G L 21$, which was shown to interact with $C m A N R 1^{56}$, and AGL21 exerted similar effects on LR development as CmANR1 $1^{27,56}$. The formation of ANR1/AGL21 heterodimers may then collectively regulate LR development in chrysanthemum. Finally, a robust root system developed in parallel with thriving shoot development and growth in chrysanthemum (Supplementary Table 2).

\section{Acknowledgements}

We appreciate Professor Yu-Jin Hao (Shandong Agricultural University, China) for kindly providing all vectors as well as EMSA and ChIP-gPCR methods that were used in this study. Meanwhile, we thank Professor Jun-Ping Gao and Professor Nan-Ma (China Agricultural Universtiy, China) for kindly supporting the wild-type tissue-cultured chrysanthemum "Iinba" as well as the protocol of chrysanthemum leaf disc transformation method. This work was supported by grants from the National Natural Science Foundation of China (31601728), Shandong Province (ZR2016CQ13), Young Scientists Funds of Shandong Agricultural University $(564024,24024)$.

\section{Conflict of interest}

The authors declare that they have no conflict of interest.

\section{Publisher's note}

Springer Nature remains neutral with regard to jurisdictional claims in published maps and institutional affiliations.

Supplementary Information accompanies this paper at (https://doi.org/ 10.1038/s41438-018-0061-y).

Received: 5 March 2018 Revised: 23 May 2018 Accepted: 28 May 2018 Published online: 01 October 2018

\section{References}

1. Nibau, C., Gibbs, D. J. \& Coates, J. C. Branching out in new directions: the control of root architecture by lateral root formation. New Phytol. 179, 595-614 (2008).

2. Giehl, R. F. \& von Wirén, N. Root nutrient foraging. Plant Physiol. 166, 509-517 (2014).

3. Bellini, C., Pacurar, D. I. \& Perrone, I. Adventitious roots and lateral roots: similarities and differences. Annu. Rev. Plant. Biol. 65, 639-666 (2014).

4. Coudert, Y. et al. Genetic control of root development in rice, the model cereal. Trends Plant. Sci. 15, 219-226 (2010).

5. Yu, P., Gutjahr, C., Li, C. J. \& Hochholdinger, F. Genetic control of lateral root formation in Cereals. Trends Plant. Sci. 21, 11 (2016).

6. Hochholdinger, F. et al. From weeds to crops: genetic analysis of root development in cereals. Trends Plant. Sci. 9, 42-48 (2004).

7. Geiss, G., Gutierrez, L. \& Bellini, C. Adventitious root formation: new insights and perspectives. Annu. Rev. Plant. Biol. 37, 127-156 (2009).

8. Li, S. W. et al. Mediators, genes and signaling in adventitious rooting. Bot. Rev. 75, 230-247 (2009).

9. Casimiro, I. et al. Auxin transport promotes Arabidopsis lateral root initiation. Plant Cell 13, 843-852 (2001)

10. Fukaki, H., Okushima, Y. \& Tasaka, M. Auxin-mediated lateral root formation in higher plants. Int. Rev. Cytol. 256, 111-137 (2007).

11. Gutierrez, L. et al. Auxin controls Arabidopsis adventitious root initiation by regulating jasmonic acid homeostasis. Plant Cell 24, 2515-2527 (2012).

12. Pacurar, D. I. et al. Auxin is a central player in the hormone cross-talks that control adventitious rooting. Physiol. Plant. 151, 83-96 (2014).

13. Du, Y. J. \& Scheres, B. Lateral root formation and the multiple roles of auxin. J. Exp. Bot. 69, 155-167 (2018).

14. Niemi, K. et al. Ectomycorrhizal fungi and exogenous auxins influence root and mycorrhiza formation of Scots pine hypocotyl cuttings in vitro. Tree Physiol. 22, 1231-1239 (2002)

15. Wynne, J. \& McDonald, M. Adventitious root formation in woody plant tissue: influence of light and indole-3-butyric acid (IBA) on adventitious root induction in Betula pendula. In. Vitr. Cell Dev. Biol. Plant 38, 210-212 (2002).

16. Lanteri, M. L., Pagnussat, G. C. \& Lamattina, L. Calcium and calcium-dependent protein kinases are involved in nitric oxide- and auxin-induced adventitious root formation in cucumber. J. Exp. Bot. 57, 1341-1351 (2006).

17. Yang, J. et al. OsCBL1 modulates lateral root elongation in rice via affecting endogenous indole-3-acetic acid biosynthesis. JGG 42, 331-334 (2015).

18. Gibbs, D. J. et al. AtMYB93 is a novel negative regulator of lateral root development in Arabidopsis. New Phytol. 203, 1194-1207 (2014).

19. Lucas, M. et al. SHORT-ROOT regulates primary, lateral, and adventitious root development in Arabidopsis. Plant Physiol. 155, 384-398 (2011). 
20. Zhao, Y. et al. The interaction between rice ERF3 and WOX11 promotes crown root development by regulating gene expression involved in cytokinin signaling. Plant Cell 27, 2469-2483 (2015).

21. Negi, S., Ivanchenko, M. G. \& Muday, G. K. Ethylene regulates lateral root formation and auxin transport in Arabidopsis thaliana. Plant J. 55, 175-187 (2008).

22. Ivanchenko, M. G., Muday, G. K. \& Dubrovsky, J. G. Ethylene-auxin interactions regulate lateral root initiation and emergence in Arabidopsis thaliana. Plant J. 55, 335-347 (2008).

23. Mauriat, M. et al. Gibberellins inhibit adventitious rooting in hybrid aspen and Arabidopsis by affecting auxin transport. Plant J. 78, 372-384 (2014).

24. Feng, S. H. et al. Arabidopsis CAND1, an unmodified CUL1-interacting protein, is involved in multiple developmental pathways controlled by ubiquitin/ proteasome-mediated protein degradation. Plant Cell 16, 1870-1882 (2004).

25. Gutierrez, L. et al. Phenotypic plasticity of adventitious rooting in Arabidopsis is controlled by complex regulation of AUXIN RESPONSE FACTOR transcripts and microRNA abundance. Plant Cell 21, 3119-3132 (2009).

26. Lavenus, J. et al. Lateral root development in Arabidopsis: fifty shades of auxin. Trends Plant Sci. 18, 450-458 (2013)

27. $\mathrm{Yu}, \mathrm{L}$. H. et al. MADS-box transcription factor $A G L 21$ regulates lateral root development and responds to multiple external and physiological signals. Mol. Plant 7, 1653-1669 (2014).

28. Benková, E. et al. Local, efflux-dependent auxin gradients as a common module for plant organ formation. Cell 115, 591-602 (2003).

29. Swarup, R. \& Bennett, M. Auxin transport: the fountain of life in plants? Dev. Cell 5, 824-826 (2003)

30. Rashotte, A. M. et al. Basipetal auxin transport is required for gravitropism in roots of Arabidopsis. Plant Physiol. 122, 481-490 (2000).

31. Yang, Y. et al. High-affinity auxin transport by the AUX1 influx carrier protein. Curr. Biol. 16, 1123-1127 (2006).

32. Swarup, R. \& Péret, B. AUX/LAX family of auxin infux carriers-an overview. Front. Plant Sci. 3, 225 (2012)

33. Weijers, D. et al. Maintenance of embryonic auxin distribution for apical-basal patterning by PIN FORMED-dependent auxin transport in Arabidopsis. Plant Cell 17, 2517-2526 (2005).

34. Wisniewska, J. et al. Polar PIN localization directs auxin flow in plants. Science 312, 883 (2006)

35. Gälweiler, L. et al. Regulation of polar auxin transport by AtPIN1 in Arabidopsis vascular tissue. Science 282, 2226-2230 (1998)

36. Blilou, I. et al. The PIN auxin efflux facilitator network controls growth and patterning in Arabidopsis roots. Nature 433, 6 (2005).

37. Feraru, E. \& Friml, J. PIN polar targeting. Plant Physiol. 147, 1533-1559 (2008).

38. Dal Bosco, C. et al. The endoplasmic reticulum localized PIN8 is a pollenspecific auxin carrier involved in intracellular auxin homeostasis. Plant J. $\mathbf{7 1}$ 860-870 (2012).

39. Ding, Z. et al. ER-localized auxin transporter PIN8 regulates auxin homeostasis and male gametophyte development in Arabidopsis. Nat. Commun. 3, 941 (2012).

40. Sawchuk, M. G., Edgar, A. \& Scarpella, E. Patterning of leaf vein networks by convergent auxin transport pathways. PLoS Genet. 9, e1003294 (2013).

41. Ganguly, A. et al. Functional analysis of the hydrophilic loop in intracellular trafficking of Arabidopsis PIN-FORMED proteins. Plant Cell 26, 1570-1585 (2014).

42. Simon, S. et al. PIN6 auxin transporter at endoplasmic reticulum and plasma membrane mediates auxin homeostasis and organogenesis in Arabidopsis. New Phytol. 211, 65-74 (2016).

43. $\mathrm{Xu}, \mathrm{M}$. et al. A PIN1 family gene, OsPIN1, involved in auxin-dependent adventitious root emergence and tillering in rice. Plant Cell Physiol. 46, 1674-1681 (2005).

44. Chen, Y. N. et al. Over-expression of OsPIN2 leads to increased tiller numbers, angle and shorter plant height through suppression of OsLAZY1. Plant Biotechnol. J. 10, 139-149 (2012).

45. Ding, Z. J. et al. Transcription factor WRKY46 modulates the development of Arabidopsis lateral roots in osmotic/salt stress conditions via regulation of ABA signaling and auxin homeostasis. Plant J. 84, 56-59 (2015).

46. Zhu, L. et al. Chrysanthemum transcription factor CmLBD1 direct lateral root formation in Arabidopsis thaliana. Sci. Rep. 6, 20009 (2015).

47. Michaels, S. D. et al. AGL24 acts as a promoter of flowering in Arabidopsis and is positively regulated by vernalization. Plant J. 33, 867-874 (2003).

48. de Folter, S. et al. A Bsister MADS-box gene involved in ovule and seed development in petunia and Arabidopsis. Plant J. 47, 934-946 (2006).
49. Dong, T. et al. A tomato MADS-box transcription factor, SIMADS1, acts as a negative regulator of fruit ripening. Plant Physiol. 163, 1026-1036 (2013).

50. Huang, B. W. et al. Overexpression of the class D MADS-box gene SI-AGL11 impacts fleshy tissue differentiation and structure in tomato fruits. J. Exp. Bot 68, 4869-4884 (2017).

51. Zhang, H. \& Forde, B. G. An Arabidopsis MADS box gene that controls nutrient induced changes in root architecture. Science 16, 407-409 (1998).

52. Tapia-Lopez, R. et al. An AGAMOUS-related MADS-box gene, XAL1 (AGL12), regulates root meristem cell proliferation and flowering transition in Arabidopsis. Plant Physiol. 146, 1182-1192 (2008).

53. Garay-Arroyo, A. et al. The MADS transcription factor XAL2/AGL14 modulates auxin transport during Arabidopsis root development by regulating PIN expression. EMBO J. 32, 2884-2895 (2013).

54. Yu, C. Y. et al. MADS-box transcription factor OsMADS25 regulates root development through affection of nitrate accumulation in rice. PLoS One $\mathbf{8}$, e0135196 (2015).

55. Liu, W. L. et al. A novel sucrose-regulatory MADS-box transcription factor GmNMHC5 promotes root development and nodulation in soybean (Glycine max [L.] Merr.). Int. J. Mol. Sci. 16, 20657-20673 (2015).

56. Sun, C. H. et al. Chrysanthemum MADS-box transcription factor CmANR1 modulates lateral root development via homo-/heterodimerization to influence auxin accumulation in Arabidopsis. Plant Sci. 266, 27-36 (2017).

57. Hong, B. et al. Expression of Arabidopsis DREB1A gene in transgenic chrysanthemum enhances tolerance to low temperature. J. Hortic. Sci. Biotechnol. 81, 1002-1008 (2006)

58. Grabherr, M. G. et al. Full-length transcriptome assembly from RNA-Seq data without a reference genome. Nat. Biotechnol. 29, 644-652 (2011).

59. Zheng, Y. et al. iAssembler: a package for de novo assembly of Roche-454/ Sanger transcriptome sequences. BMC Bioinforma. 12, 453 (2011).

60. Yang, Y. J. et al. A zinc finger protein regulates flowering time and abiotic stress tolerance in chrysanthemum by modulating gibberellin biosynthesis. Plant Cell 26, 2038-2054 (2014).

61. Karp, P. D., Paley, S. \& Romero, P. The pathway tools software. Bioinformatics 18, S225-S232 (2002).

62. $\mathrm{Xu}, \mathrm{Y}$. J. et al. Transcriptome sequencing and whole genome expression profiling of chrysanthemum under dehydration stress. BMC Genom. 14, 662 (2013).

63. Hu, D. G. et al. MdMYB1 regulates anthocyanin and malate accumulation by directly facilitating their transport into vacuoles in apples. Plant Physiol. 170, 1315-1330 (2016)

64. Chen, Q. et al. The basic helix-loop-helix transcription factor MYC2 directly represses PLETHORA expression during jasmonate-mediated modulation of the root stem cell niche in Arabidopsis. Plant Cell 23, 3335-3352 (2011).

65. An, J. P. et al. The bZIP transcription factor MdHY5 regulates anthocyanin accumulation and nitrate assimilation in apple. Hort. Res. 4, 17023 (2017).

66. Lin, W. H. et al. At5PTase13 modulates cotyledon vein development through regulating auxin homeostasis. Plant Physiol. 139, 1677-1691 (2005).

67. Wynne, J. \& Treisman, R. SRF and MCMI have related but distinct DNA binding specificities. Nucleic Acids Res. 20, 3297-3303 (1992).

68. Lee, $\mathrm{H}$. et al. The AGAMOUS-LIKE 20 MADS domain protein integrates floral inductive pathways in Arabidopsis. Genes Dev. 14, 2366-2376 (2000)

69. Theißen, G. Development of floral organ identity: stories from the MADS house. Curr. Opin. Plant Biol. 4, 75-85 (2001)

70. Ripoll, J. J. et al. A novel role for the floral homeotic gene APETALA2 during Arabidopsis fruit development. Development 138, 5167-5176 (2011).

71. Koo, S. C. et al. Control of lateral organ development and flowering time by the Arabidopsis thaliana MADS-box gene AGAMOUS-LIKE6. Plant J. 62, 807-816 (2010).

72. Sang, X. C. et al. CHIMERIC FLORAL ORGANS1, encoding a monocot-specific MADS box protein, regulates floral organ identity in rice. Plant Physiol. 160 788-807 (2012)

73. Tripathi, V. et al. CIPK6, a CBL-interacting protein kinase is required for development and salt tolerance in plants. Plant J. 58, 778e790 (2009).

74. Robles, L., Stepanova, A. \& Alonso, J. Molecular mechanisms of ethylene-auxin interaction. Mol. Plant 6, 1734-1737 (2013).

75. Tsuchisaka, A. \& Theologis, A. Unique and overlapping expression patterns among the Arabidopsis 1-amino-cyclopropane-1-carboxylate synthase gene family members. Plant Physiol. 136, 2982-3000 (2004).

76. Rahman, A. et al. Auxin is a positive regulator for ethylene-mediated response in the growth of Arabidopsis roots. Plant Cell Physiol. 42, 301-307 (2001). 
77. Stepanova, A. N. et al. Multilevel interactions between ethylene and auxin in Arabidopsis roots. Plant Cell 19, 2169-2185 (2007).

78. Ruzicka, K. et al. Ethylene regulates root growth through effects on auxin biosynthesis and transport-dependent auxin distribution. Plant Cell 19 2197-2212 (2007).

79. Lewis, D. R. et al. Ethylene inhibits lateral root development, increases IAA transport and expression of PIN3 and PIN7 auxin efflux carriers. Development 138, 3485-3495 (2011).

80. Cai, X. T. et al. Arabidopsis ERF109 mediates cross-talk between jasmonic acid and auxin biosynthesis during lateral root formation. Nat. Commun. 5, 5833 (2014).
81. Staswick, P. E., Su, W. \& Howell, S. H. Methyl jasmonate inhibition of root growth and induction of a leaf protein are decreased in an Arabidopsis thaliana mutant. Proc. Natl. Acad. Sci. USA 89, 6837-6840 (1992).

82. Remans, T., Nacry, P. \& Pervent, M. The Arabidopsis NRT1.1 transporter participates in the signaling pathway triggering root colonization of nitrate-rich patches. Proc. Natl. Acad. Sci. USA 103, 19206-19211 (2006).

83. Ho, C. H. et al. CHL1 functions as a nitrate sensor in plants. Cell 138, 1184-1194 (2009). 